\title{
BMJ Open My child's legacy: a mixed methods study of bereaved parents and providers' opinions about collaboration with NICU teams in quality improvement initiatives
}

To cite: Bourque CJ, Dahan S, Mantha G, et al. My child's legacy: a mixed methods study of bereaved parents and providers' opinions about collaboration with NICU teams in quality improvement initiatives. BMJ Open 2020;10:e034817. doi:10.1136/ bmjopen-2019-034817

- Prepublication history for this paper is available online. To view these files, please visit the journal online (http://dx.doi. org/10.1136/bmjopen-2019034817).

Received 12 October 2019 Revised 22 May 2020 Accepted 13 July 2020

\section{Check for updates}

(c) Author(s) (or their employer(s)) 2020. Re-use permitted under CC BY-NC. No commercial re-use. See rights and permissions. Published by BMJ.

${ }^{1} \mathrm{CHU}$ Sainte-Justine Research Center, CHU Sainte-Justine, Montréal, Quebec, Canada ${ }^{2}$ Pediatrics, Université de Montréal, Montreal, Quebec, Canada

${ }^{3}$ Division of Neonatology, CHU Sainte-Justine, Montréal,

Quebec, Canada

${ }^{4}$ Prema-Québec, Longueuil, Quebec, Canada

${ }^{5}$ Québec Research Network on Palliative and End-of-Life Care, RQSPAL, Montréal, Québec, Canada

Correspondence to Dr Claude Julie Bourque; claude.julie.bourque@ umontreal.ca

\section{ABSTRACT}

Objective Although stakeholders' participation in healthcare is increasingly recommended, bereaved parents are often excluded for perceived potential risks to them. The objective of this study is to describe the ongoing involvement and the perspectives of bereaved parents engaged in different types of activities in Neonatal Intensive Care Units and providers who work with them.

Design/methods Mixed methods convergent analysis. Setting Canadian paediatric tertiary care university hospital.

Participants All bereaved members of the resource parents group ( $\mathrm{n}=8$ ) and most providers who work with them $(n=16)$ answered a satisfaction/needs questionnaires.

Results Since 2011, eight bereaved parents were involved in a large number of activities mostly related to palliative care (research, education or clinical care initiatives). Three engaged in peer-to-peer support activities while the others preferred activities outside of clinical units and/or without direct interactions with other families. All of them reported that their participation had positive impacts, but two parents also reported a reactivation of traumatic experiences during a medical simulation activity. All participants expressed a desire for further collaboration. Motivation to contribute gravitated around two central themes: helping others and helping themselves. Many wanted to give back, help other families, improve the system and meet with providers who had cared for their child. All stated that this kind of involvement empowered them and gave meaning to their experiences. Providers and researchers all reported positive experiences, mainly due to the unique perspectives of bereaved parents who took part in their projects.

Conclusion(s) With careful recruitment and supervision, some bereaved parents can become resource parents involved in different types of activities. It is important to understand the positive impacts this type of engagement can have on their healing process and to control the risks related to their participation. Research is needed to develop pertinent tools and measures to evaluate the outcomes and impacts of their participation.
Strengths and limitations of this study

- This is the first study, to our knowledge, to inves tigate the views of both healthcare providers and bereaved parents on their engagement in different types of initiatives in paediatrics.

- Almost all stakeholders (parents, providers and researchers) involved in the activities participated in the study $(96 \%, n=23 / 24)$.

- One experienced resource parent was involved as co-investigator in the design of the study, the conception of tools, data analysis (including coding qualitative data) as well as writing and dissemination activities.

- This was originally an internal quality control and satisfaction clinical study that raised so much interest that the authors decided to publish the most transferable results.

- Limitations of this study include the representation of one site and one paediatric service only as well as the small number of parents, the latter being partially compensated by the variety and duration of their experiences as resource parents.

\section{INTRODUCTION}

Almost all neonatal deaths in industrialised countries occur in Neonatal Intensive Care Units (NICU), ${ }^{1}{ }^{2}$ with an important number originating from end-of-life decisions made by clinicians and parents, for whom these choices are often the most difficult of their life. ${ }^{3} 4$ 'NICU parents' are at risk of adverse outcomes, such as post-traumatic stress disorder and depression. ${ }^{356}$ Despite lasting impacts, the majority of bereaved parents eventually find some healing and many report post-traumatic growth. ${ }^{78}$

Many interventions have been attempted to improve the well-being of families in the NICU, such as involving veteran parents. ${ }^{9}$ In our definition, resource parents are parents who previously experienced the hospitalisation of their child in NICU and use that 
unique perspective to improve care. ${ }^{10-12}$ Because they are often considered part of a vulnerable population, bereaved parents are often excluded from this type of collaboration for ethical and practical reasons, mainly potential risks to their well-being. ${ }^{1013} 14$ Yet, their perspectives and opinions are recognised as important and are increasingly sought. ${ }^{15-19}$

Since 2011, we have teamed up with more than 30 resource parents-some of which are bereaved parents-as part of many different initiatives. ${ }^{10} 12{ }^{20} \mathrm{We}$ wished to expand our programme and recruit more bereaved parents, but wanted to ensure that we would do so in a responsible fashion. After almost a decade of experience, we have done a rigorous, in depth review of our programme. The goals of this study were to (1) describe the variety of activities in which bereaved resource parents participate, (2) describe the perspectives of bereaved parents and the clinicians who work with them and (3) propose practical recommendations for those who wish to take part in such initiatives. This research is part of a quality improvement clinical programme. This broader project uses multiple and mixed methods approaches for evaluation, measurement of impacts, unmet needs analysis and satisfaction surveys. The results are used for evidence-based orientation and decisions on support programmes for patients. ${ }^{21} 22$ The specific results presented in this article are strictly about the participation of bereaved resource parents and come from a study that was done in preparation of new activities to support the decision regarding whether or not to recruit and involve them in the development and animation of a new peer-to-peer workshop programme.

\section{METHODS}

\section{Setting and participants}

The PAF (Partenariat Famille) team was created in 2011 to recruit, integrate and coordinate resource parents to optimise neonatal initiatives in Sainte-Justine University Health Center, a mother-child tertiary care universityaffiliated hospital. The NICU has 70 beds and admits on average 1100 neonates a year, with about 50 to 60 annual deaths. Resource parents were recruited among former parents either because they came forward to participate or were suggested by providers.

Bereaved resource parents were recruited a minimum of 2 years after their child's death, and were offered compensation to participate (transport, parking, meals). For complex initiatives, they received specific detailed training and preparation. For example, when involved in peer-to-peer support, they followed the same training as other volunteers and received additional support. On the other hand, for many simple initiatives, such as giving their opinion on a parent-participant consent form, they did not require specific training. More details on the recruitment of resource parents and their gradual integration and training are available in our previous publications. ${ }^{10} 12$

\section{Data collection}

Qualitative field documentation

All the field notes, correspondence and reports pertaining to activities performed by bereaved resource parents since 2011 were reviewed and sorted by the principal investigator (AJ). The initiatives were categorised by the analysts as either research, clinical or teaching and they were classified further as taking place in hospital setting or not and as involving or not direct interactions with hospitalised families or providers. For example, a bereaved resource parent who has co-taught a palliative care course three times would be analysed as having participated in one teaching initiative, and in a total of three activities taking place at the hospital and in interaction with providers only.

\section{Mixed questionnaires}

From January to April 2017, an online questionnaire was distributed to all resource parents, bereaved or not, who had been involved in neonatal initiatives, requesting feedback on their experience $(n=22$ at the time, $100 \%$ participation, three of them by telephone interviews with the principal investigator $(\mathrm{AJ})$ who recruited and worked with them). The questionnaire included closed questions on their preferences regarding past and future activities (types of activities, setting, availability, etc) and open questions that invited them to describe, in their own words, their motivations, their positive and negative experiences, their opinion on compensation and their views on the impacts of their participation. For the purpose of this study, we limited our analysis to the answers of the bereaved resource parents.

A different online questionnaire was sent to the 17 providers who collaborated with bereaved resource parents. They were asked close-ended questions to categorise their experience with resource parents as well as open-ended question on their opinions regarding the positive and negative aspects of their collaborations and the impact of the parents' participation in their activities.

\section{Data analysis}

We used descriptive statistics for quantitative data (absolute and relative frequencies) from our compilation on activities and participation and from quantitative items in the questionnaire. Qualitative data from the openended questions in the questionnaire were analysed using a descriptive and inductive content analysis with NVivo 11.4.1 software package. ${ }^{23-26} \mathrm{~A}$ descriptive approach was used to develop and define emerging themes and subthemes by a team of four investigators (one trained resource parent, two neonatologists and one methodologist). All qualitative content was double coded in an independent fashion. Reliability and stability of the coding process was assessed systematically, and discrepancies were resolved through discussion between investigators to 
reach consensus. Both quantitative and qualitative results are combined and presented in a convergent manner by merging and embedding them as often done in case studies. ${ }^{21} 2227$

\section{Patient and public involvement statement}

The study was conducted with an experienced resource parent acting as co-author (GM). She was involved in all aspects of the study and for its entire duration, from design to tools development, data analysis, writing and dissemination plan.

\section{Ethical considerations}

This project was done in the context of an internal programme evaluation study. The Research Ethics Committee of the CHU Sainte-Justine Research Center believed it did not require an ethical review and expressed no objection to the publication of the study, as it was done to inform the preparation of a quality improvement clinical study involving bereaved parents. Participants provided informed consent at the beginning of the online questionnaire (implied consent by accepting to answer the questionnaire) once they had read the introduction, which explained the study and informed them that the data created would be coded and that their identity would not be shared.

\section{RESULTS}

At the time of the survey (winter 2017), eight bereaved resource parents had been collaborating with providers for at least 2 years. Their demographic profile and details about their experience in the NICU are summarised in table 1 . The providers $(n=16 / 17)$ included nine neonatologists, two nurses, two fellows and three consultant nurses (nutrition, breastfeeding).

\section{Description of activities}

The compilation of our field documentation shows that since 2011, bereaved resource parents were involved in a total of 465 activities categorised in 17 types of initiatives in three broader categories: research, education and clinical care or administration (table 2).

All bereaved resource parents collaborated to optimise clinical care, and six were also involved in research and education. They had various degree of participation: from a minimum of one activity in one initiative to a maximum of 167 activities in 17 different types of initiatives over the period. Not all these partnership activities required them to be physically present in the hospital or to be in direct contact with parents or patients. For example, they could review information given to parents from home. Other activities were more complex and required contact with bereaved families, for example, 'matched buddies' for 'new' bereaved parents (table 2). Most research activities occurred outside of the hospital setting, for example, reviewing consent forms or research protocols (table 2). Educational activities often required
Table 1 Characteristics of bereaved resource parents and their children

\begin{tabular}{ll}
\hline Bereaved resource parents $(\mathbf{n}=\mathbf{8})$ \\
\hline Gender & $\begin{array}{l}\text { Five mothers, } 3 \text { fathers (two } \\
\text { couples) }\end{array}$ \\
\hline $\begin{array}{l}2 \times \text { high school } \\
6 x \text { college/university }\end{array}$ \\
$\begin{array}{ll}\text { Science, engineering, public } \\
\text { or retail management, law, } \\
\text { education, healthcare, public } \\
\text { service, home parent }\end{array}$ \\
$\begin{array}{ll}\text { First time parents } & \mathrm{n}=5\end{array}$ \\
$\begin{array}{l}\text { Experienced multiple birth and } \\
\text { discordant survival }\end{array}$
\end{tabular}
discordant survival

Age of parent at child's death Min: 28; max: 43

Babies of bereaved resource parents hospitalised in NICU who died $(n=9)^{*}$

\begin{tabular}{|c|c|}
\hline Year of birth/death of babies & 2005 to 2014 \\
\hline \multirow[t]{3}{*}{ Type of birth } & $2 \mathrm{x}$ triplets \\
\hline & $2 x$ twins \\
\hline & $5 x$ singletons \\
\hline \multirow[t]{2}{*}{ Gestational age (in weeks) } & $1 \times 39$ weeks \\
\hline & $8 x$ less than 28 weeks \\
\hline \multirow{3}{*}{$\begin{array}{l}\text { Length of hospital stay before } \\
\text { death (deaths all occurred in } \\
\text { the NICU) }\end{array}$} & $1 \mathrm{x}>3$ months \\
\hline & $1 \times 1-3$ months \\
\hline & $7 x<1$ month \\
\hline $\begin{array}{l}\text { Number of babies who had } \\
\text { surgery }\end{array}$ & 3 \\
\hline \multirow[t]{5}{*}{$\begin{array}{l}\text { Main diagnosis leading to } \\
\text { death }\end{array}$} & $\begin{array}{l}8 \times \text { complications associated } \\
\text { with prematurity }\end{array}$ \\
\hline & $3 x$ necrotising enterocolitis \\
\hline & $3 \times$ sepsis \\
\hline & $2 x$ pulmonary insufficiency \\
\hline & $1 \mathrm{x}$ congenital anomaly \\
\hline
\end{tabular}

*One participating parent lost two babies, which explains the difference in numbers (eight parents and nine babies) NICU, Neonatal Intensive Care Unit.

coming to the hospital and being in contact with students and trainees for co-teaching, testimonials, feedback and evaluation during medical simulation sessions, classes or case-based learning (table 2).

Although most of the activities were related to palliative care and bereavement, four bereaved resource parents also participated in activities that were not associated with these topics, such as being involved in a quality control committee to improve clinical care. One father specifically stated that he did not want to be involved in projects pertaining to death, as illustrated in this quote from the correspondence notes: "I want to improve the care babies and parents get, give back, focus on the positive, life, give what I can give. But I don't want to talk about death and things related to that". 
Table 2 Types of initiatives involving bereaved resource parents

\begin{tabular}{|c|c|c|}
\hline Types of initiatives & $\begin{array}{l}\text { Total number of } \\
\text { activities }\end{array}$ & $\begin{array}{l}\text { Number of BRPs } \\
\text { involved }\end{array}$ \\
\hline \multicolumn{3}{|l|}{ Research } \\
\hline Presentation at conferences, panels (testimonial, workshops, study results) & 24 & 3 \\
\hline Co-redaction, co-author, sole author of articles or abstracts & 52 & 4 \\
\hline $\begin{array}{l}\text { Co-creation, review, editing research protocols, production of research tools, reviewing } \\
\text { and co-creating consent forms }\end{array}$ & 46 & 4 \\
\hline Member of Parent Advisory Board, member of Data Safety and Monitoring Committee & 8 & 1 \\
\hline Collaboration in the recruitment of families, data acquisition & 6 & 4 \\
\hline \multicolumn{3}{|l|}{ Education } \\
\hline Testimonials and narratives integrated in courses & 18 & 1 \\
\hline $\begin{array}{l}\text { Co-teaching with providers and educators, co-evaluation of trainees during simulations, } \\
\text { parental presence/feedback during simulations (workshops, case-based learning) }\end{array}$ & 8 & 3 \\
\hline Staff/interdisciplinary meetings and presence at clinical care committees & 51 & 2 \\
\hline Welcome visit for new parents & 37 & 2 \\
\hline $\begin{array}{l}\text { Creation, evaluation and dissemination of information resources for new parents (and } \\
\text { bereaved parents) }\end{array}$ & 7 & 5 \\
\hline $\begin{array}{l}\text { Involvement in the coordination of resource-parent programme: matching provider's } \\
\text { projects with selected parents, recruitment, training and support of new resource parents }\end{array}$ & 42 & 3 \\
\hline $\begin{array}{l}\text { Hospital design/redesign, development of clinical care protocols, quality control projects, } \\
\text { evaluation of clinical care }\end{array}$ & 32 & 4 \\
\hline 'Matched buddies' for new bereaved parents (including contacts via internet or phone) & 28 & 3 \\
\hline
\end{tabular}

BRPs, bereaved resource parents; NICU, Neonatal Intensive Care Unit.

\section{Bereaved parents' perspectives}

In the parents' questionnaire, most bereaved resource parents expressed that they wanted to be/continue being in contact with providers, students and bereaved families (table 3). Only three parents wanted to participate in activities where they would be in direct contact with parents of a sick baby, and two reported not wanting to be in contact with the clinical environment. The correspondence notes include this representative quote taken from a father's message: 'I want to continue helping, but I don't want to go in that unit again, smell the hand wash, hear the bells".

(1) Motivations, experience and perceived impacts

In the questionnaire, parents were asked about the circumstances of their child or children's death, and their answers reflect both different and similar experiences. Four parents gave factual data only (child's sex, diagnosis and age at death): "Twin boy died from necrotising enterocolitis at 2 weeks". Three parents added more details and recounted the decision to withdraw lifesustaining interventions, including this couple, who show a lot of coherence when describing their experience in their own words:

'Her lungs were very sick and she was not breathing on her own. The doctors suggested we remove the breathing tube because the damage was very important.' (mother)

'She had several complications during her stay. Her condition (...) did not allow her to continue her battle. So we ended it.' (father)

We noted that this couple shared the same story, but had very contrasted preferences regarding the types of activities they were interested in.

When asked about their positive and negative experiences as well as their perceived impacts of their participation, all parents had positive comments. After identifying specific themes in the initial coding of the answers to these three questions, it became obvious that they all related to two general overarching themes: (a) helping others $(8 / 8)$ and (b) helping themselves $(7 / 8)$. The general themes, 
Table 3 Preferences of bereaved resource parents for activities in palliative care

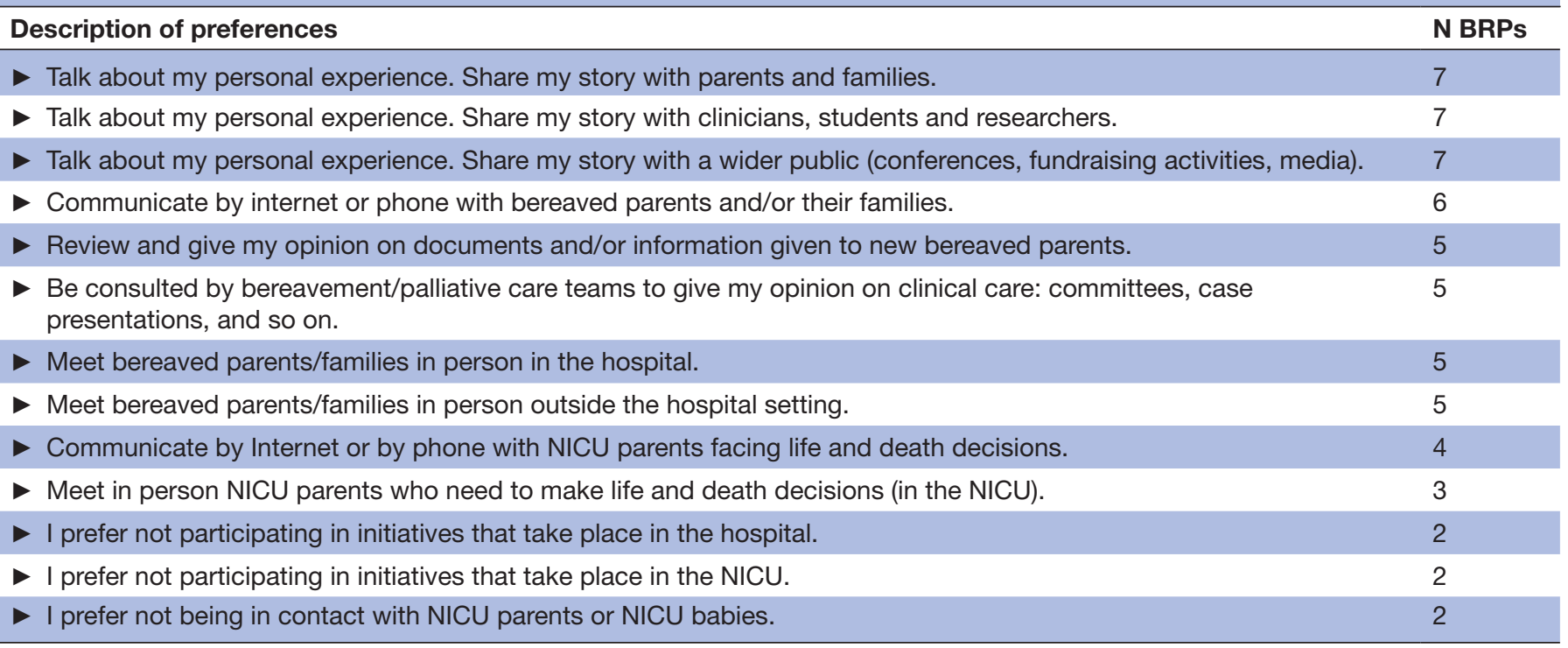

BRPs, bereaved resource parents; NICU, Neonatal Intensive Care Unit.

specific themes and representative quotes are presented in table 4.

Overall, parental responses illustrate that they believed their contribution - and thus the help it brought - originated from their desire to share the legacy of their child's life, as well as their experience as parents. Although we aimed to keep the analysis at a very descriptive level, a more comprehensive analysis revealed this notion of legacy as a central theme, which we introduced in the title. This also strongly resonated with the parents, to whom we presented the preliminary results of this analysis. Through their engagement, they wished-and often felt some kind of responsibility to-keep the memory of their child alive and to make sense of their journey by putting their experience and knowledge to good use. Terms used in the majority of their answers to open-ended questions include, for example, 'hope to give a voice to parents in my situation', 'give meaning to the experience we went through', 'doing this in the memory of my child', 'giving back since we had the chance to meet wonderful people through our ordeal', 'my child enabled this, made it possible', 'share with families who went through the same difficult situations', 'give me some kind of control that I did not have during [my child's hospitalisation', and so on.

Only one parent reported a negative experience, a mother who was invited, with others, to give her opinion on the new unit to ensure it was safe for patients and optimal for family-integrated care. In this context, she participated in a unit simulation, playing the role of the mother at the bedside of a manikin baby. Although she was not involved in a mock simulation of a distressful intensive intervention, there were more intense scenarios in the ward and the general ambience of the unit, with the bells and staff, brought back painful memories in the following days. In the satisfaction questionnaire completed a few weeks after the activity, this mother shared her thoughts on her negative experience:

I found it difficult, during simulations. It somehow made me relive parts of the experience of my baby's death. I really wanted to participate. I would go so far as to say that I felt the need to. However, my reactions are a little difficult to predict, even for me! I do not know how much it will benefit me to immerse myself too deeply in my memories... one thing is certain, I will continue helping as much as I can, while making sure to preserve my mental health.

When it comes to simulations, it should be noted that notes about another mother (not bereaved) who participated in the same activity show a high level of risks. During the simulation, although she was informed about the scenarios and did not expect any problems, this very experienced resource parent became very emotional, remembering intense moments shared with her children's father in NICU more than 10 years prior. She talked about it after the activity and following some debriefing, she decided to go on with the other planned 'lighter' scenarios.

(2) Training, support and feedback

All bereaved resource parents reported in the questionnaire that they received training and preparation from the providers who invited them to participate. Four parents thought we could improve their experience by suggesting the following steps: (1) describe precisely the objectives and timeline of their involvement, (2) describe everybody's role before and if needed during the activity and (3) identify one specific provider or researcher they could contact if necessary before, during or after the activity.

Most bereaved resource parents (6/8) reported receiving support during their participation and 
Table 4 Positive experience and perceived impacts from parents

\section{Specific themes Quotes}

General theme: Helping others, making things better

Giving the family perspective (7/8) _ "I wanted to help. I can see things with the eyes of a parent." (father)

- "Participating in this research gave a voice to families who are generally not able to write in the medical literature." (mother)

- "I feel I was able to look at the research protocol through different eyes, those of a parent. I was able to give articles a parent voice." (father)

Helping bereaved parents (6/8)
Improving the system (6/8)
"We hope we have helped other families who mourn." (mother)
"I think I can also provide not just a parent's view, but a non-medical view, to improve
things. Isn't it bizarre that providers teach each other how to communicate with parents,
how to be sensitive with parents, and parents are not involved in this? They see their
world through statistics and prognosis, we see it also with our heart, with love. For
example, we do not want only to know that our child has a high percentage of death,
we want to know how this will change our life, how we will be able to face this, what this
means, in the practical sense, for our family. We can change the system a bit." (mother)
"I find it important to give because we had the chance to meet wonderful people through
this ordeal." (mother)

General theme: Helping self, making sense Meaning making (6/8)

$\rightarrow$

Memory/legacy (5/8)

Empowerment (5/8)

Repairing/reinvesting the relationship with providers (4/8)
"Participating in these activities gives sense to all the experiences we had, no matter how difficult it was. I would even say that it fills and answers a need." (mother)

- "It helps me make sense of the NICU stay, to also have something 'good' about all this experience... apart from my kids, I mean." (mother)

- "I want to do this in memory of my son." (mother)

- "This research project is a legacy from my child's life. She enabled this, she made it possible, and she made life better for other families." (mother)

- "I like feeling useful." (mother)

- "Collaborating in research projects that had to do with what my daughter had, it is some kind of control that I did not have during the hospitalisation." (father)

- "She (the neonatologist) was great and gave me hope that not all physicians treated fragile babies the way my daughter was treated.... also actually participated because she told me I knew more about parents than she did, that we could both make things better." (mother, on motivations)

"I love seeing the nurses and doctors again." (mother)

NICU, Neonatal Intensive Care Unit.

appreciated it. All wanted to receive feedback about their participation, and follow-up regarding the projects they were involved in. They want to know why involving a parent helped, how they could improve and always be informed about the research results (including articles and conferences).

(3) Remuneration and compensation

When asked about compensation (reimbursements for travelling and meals) and remuneration (additional payments), bereaved resource parents answered it depended on the nature of the activity and tasks. For short and simple activities, they did not want compensation nor remuneration. For activities that required more time and effort, three reported wanting to be compensated and remunerated and three reported compensation and remuneration should only occur if they had to travel, for example, to the hospital or a conference. Three parents answered that they would prefer to give back all or some of the money to the family-partnership programme. Three also expressed some discomfort with the question and this subject. For example, one mother wrote : 'I participate for the cause, not for the money', and one father expressed uncertainty about the subject: 'I never thought I would be paid. Well, who wouldn't want to be? I also know there is not much money in the research world... and it is a pleasure to help.'

\section{Providers' perspectives $(n=16)$}

All clinicians and researchers reported that teaming up with bereaved resource parents had positive impacts. The main themes invoked in their answers were mostly centred on the parental perspective and feedback $(n=11)$, which lead to a better understanding of the needs of families. For example, a neonatologist researcher gave concrete details about what parents can sometimes see and do better than providers and researchers: 'They can optimise a research protocol, consent forms. They also see some simple solutions in the health system and how to improve the unit through simple solutions that are not 


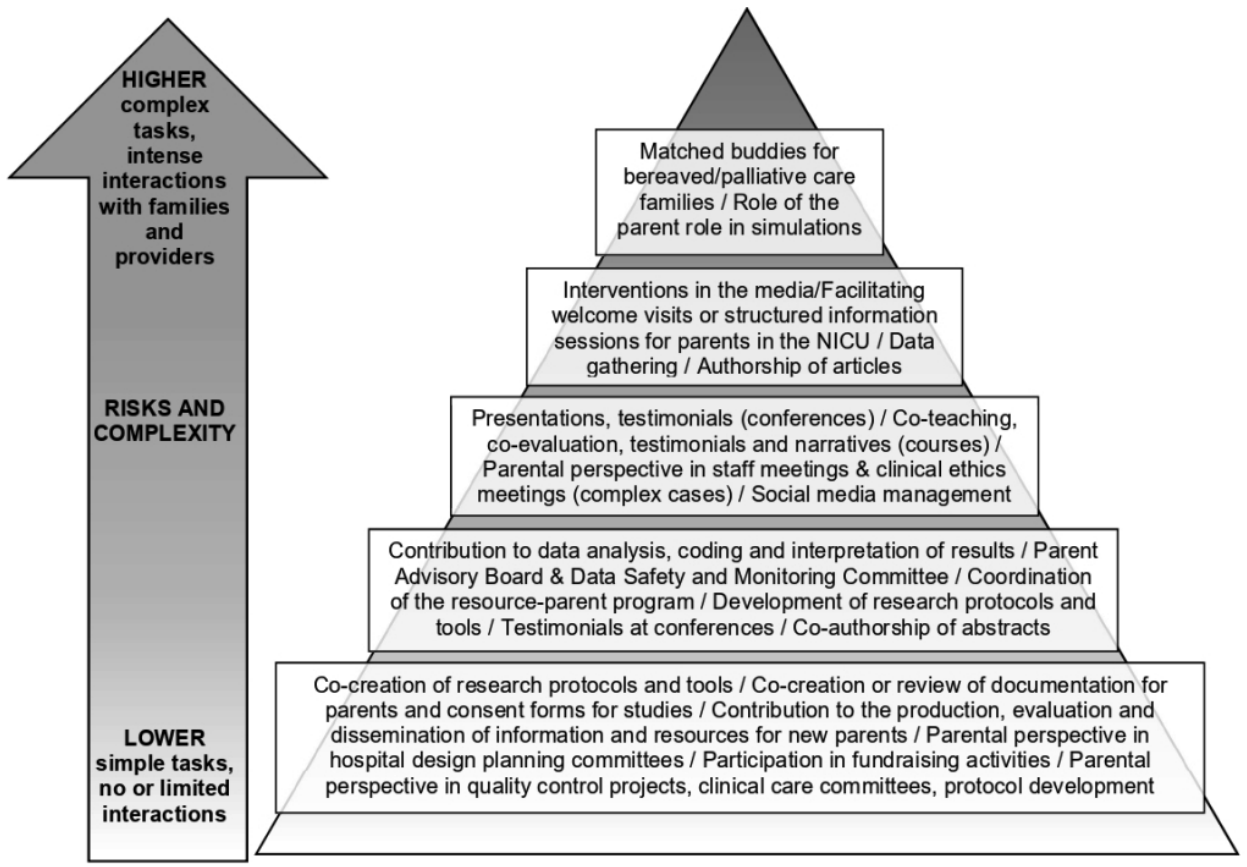

always obvious to the clinicians'. A nurse reflected on the depth of parents' contributions and feedback:

They give us a better understanding of the needs of families, how to develop meaningful relationships (less superficial or transitory).

Another neonatologist described how this type of collaboration can improve team work:

It is an enriching experience that brings a different and necessary point of view. It also allows multidisciplinary discussions to be more focussed on the patients and families. There is also more respect among members of the clinical team when parents are present.

None reported negative impacts on the involvement of bereaved resource parents but two reported challenges, such as the limited availability of resource parents and the lack of tools and guidelines on these new forms of collaboration.

\section{DISCUSSION}

Parent and patient stakeholders are essential actors that can help us improve our practices in clinical care, medical education and research. ${ }^{7} 192829$ On the other hand, bereaved parents are rarely recruited for these kinds of initiatives because they are considered a vulnerable population. To our knowledge, we are the first to report the practical aspects of integration of bereaved resource parents in many types of activities.

Parents who experienced the death of their child in NICU are all unique: some are young first-time parents while others are already parents who had a baby hospitalised in the NICU before. Many parents experienced a multiple birth and the death of one twin or triplet not long after birth. There is no other speciality in paediatrics where parents regularly experience the death of a child and the need to come back to the same intensive hospital unit to care for their other child. ${ }^{30} 31$ It is therefore crucial to take the time to talk to parents on recruiting to understand their unique story and how they feel about their involvement in the different types of activities.

Some activities are simpler and more common than others (see table 2), such as those that do not involve coming to the hospital or meeting NICU parents. As a general guide, we designed a pyramid of complexity (figure 1) to categorise the activities according to their complexity and their potential risks for parents. At the base of the pyramid are the simple, low-risk and more common activities most bereaved parents can easily do and should begin with. Not all of them will want to 'climb' the pyramid and get involved in more complex initiatives. Indeed, bereaved resource parents have different preferences regarding the type of activities they participate in and their degree of involvement. ${ }^{10}{ }^{12}$ Some only engaged in initiatives outside the hospital, possibly for time/distance constraints or because they did not wish to come back to the hospital. Some may also wish to participate in activities that are not related to palliative care and focus on improvements instead of their loss. For example, four bereaved parents who lost their child because of complications of prematurity—such as necrotising enterocolitis and/or infection-participated in quality improvements projects aimed at preventing these complications. 
Box 1 Practical recommendations for teams who wish to incorporate bereaved parents in their research, teaching or clinical activities

\section{Coordination}

- Create a multidisciplinary team consisting of one or two bereaved resource parents, at least one physician, one nurse and at least one individual with an administrative role.

Recruitment and orientation

- Recruit and work with NON bereaved stakeholders first, integrating them in simple, and then complex activities.

- Bereaved resource parents should generally be recruited at least 1 year after the death of their child.

- Ask bereaved resource parents about their motivations, their goals and interests (using practical examples). Are they able to share their story?

- Make sure they know that for some tasks, they do not have to come to the hospital and/or meet Neonatal Intensive Care Unit (NICU) parents.

- Recruit and work with parents of discordant twins or triplets (including one survivor) when starting to work with bereaved resource parents.

- It is preferable to gradually integrate bereaved parents, making them start with simple tasks (see figure 1). This will decrease the risks to them and other parents.

Training and preparation

- Give details about the project: goals, steps, duration and what is expected from them in a practical fashion.

- Inform everyone involved in the projects (staff, students, trainees) about bereaved resource parents' roles, use the name of their deceased child. They should be informed and sometimes reminded that bereaved resource parents are not members of the hospital staff nor actors.

- Make sure parents are prepared for their activity. For example, a bereaved parent who wishes to share his experience with a class of medical students could be asked to write about his experience, then speak about it with his family and the teacher of the class to better prepare.

- Greet parents when they first come to the hospital. Visit the NICU with them the first time. Some parents may then decide they do not wish to engage in activities in the NICU or with NICU parents.

Supervision/support

- Make sure bereaved resource parents have a contact person they can easily reach if they need help or information.

- Monitor how the project is going, ask them for feedback and adapt activities or tasks if needed.

Feedback and follow-up

- Offer support after the activity, especially if it was intense.

- Share information with them about results and impacts of their implication.

- Share all publications and presentations they may have contributed to. Include them as co-authors in abstracts and articles if they satisfy authorship.

- Ask them about their views on the activity, their satisfaction and their recommendations.

\section{Compensation/remuneration}

- Always offer compensation (transportation, parking, meals).

- Adapt incentives to the context and requirements of each activity. Inform parents some resource parents want compensation and others not. Offer a way they can 'waive' their compensation or give it back (receive a charity receipt, for example, when they give back).
These partnerships were described as positive by all bereaved resource parents and providers who teamed up with them. Parents reported they wanted to help others and improve care; by participating, they also gave meaning to their loss, which probably contributed to their healing process. ${ }^{78}$ Providers also reported these stakeholders enriched projects by bringing their complementary and unique perspective. Harm was reported by one participant who experienced unexpected flashbacks during a simulation with mock codes.

Post-traumatic stress symptoms are common for parents who experienced the NICU. ${ }^{6}$ In our experience, medical simulations, where parents play the role of NICU parents are high-risk situations as shown in the Pyramid of complexity where this type of activity is found at the top of the pyramid. ${ }^{12}$ As the integration of stakeholders-playing their role-seems to be increasing in medical simulations, our experience shows the existence of risks for patient or parent participants. Bereaved resource parents should be informed about these risks and made aware that they can interrupt their participation whenever they want to. The parents' willingness to participate should not be the only factor to take into consideration, as it remains the provider's or researcher's responsibility to control carefully what more fragile parents should and should not be exposed to. For example, viewing a video of a mock code, with the sound of the alarms, may help them decide if this is an activity they want to do. In all cases it is imperative to know the family story and remember that all parents are unique.

Adding the perspective of clinicians to the parents point of views was helpful, as it brought more depth to the results and anchored the recommendations we wish to share in both the parents' and providers' opinions. We have developed several practice points that may be helpful for teams who wish to embark on such initiatives with bereaved stakeholders in paediatrics (box 1). Because of knowledge gaps in this field, they should be regarded as practical suggestions that need to be evaluated and adapted to each specific project, and they could be useful for comparative analysis between organisations and countries as well as for the development of more formal guidelines.

This study has several limitations. It is limited to a single centre and did not include any outcome measures, such as clinical outcomes. We also have to keep in mind that only some bereaved parents will want to get involved in such initiatives. For this reason, this represents the perspectives of some bereaved parents, those who engage in these activities.

Although stakeholders' participation in healthcare is increasingly recommended, practical knowledge about their participation is rarely published. Bereaved parents are rarely recruited because they are considered a vulnerable population. Not all bereaved parents want to become resource parents, but by knowing their story and understanding their perspectives, it is possible to integrate them in our teams to improve clinical care, teaching 
and research. This type of collaboration is rich and gives bereaved parents an opportunity to do something positive for others and contributes to their healing process: for most of them, this is, in a way, their tribute to their child's life and legacy.

Acknowledgements The authors wish to thank the other members of the PAF team (Partenariat Famille) who made this study possible: Eliane Charbonneau, Melissa Savaria, Fanny Labelle, Josée Prince, Thuy Mai Luu and Steve Turmel. Our sincere gratitude goes to all the resource parents and patients who teamed up and continue to work with us. They teach us how to improve neonatology, make our care better and are a constant source of inspiration. We thank Keith Barrington for his careful review and help for this article.

Contributors CJB co-created the concept and design of this work, leaded the procedures for data acquisition and analysis, made a substantial contribution to data interpretation and drafted the original article. SD co-created the concept and design of this work, made a substantial contribution to data acquisition and analysis and drafted sections. GM made a substantial contribution to the design of the work and to data acquisition and analysis and help select appropriate citations. MR made a substantial contribution to the design of the work and to data acquisition and analysis. AJ co-created the concept and design of this work, made a substantial contribution to the design of the work and to data analysis and interpretation and drafted the original article. All authors revised the article critically for important intellectual content, approved the version to be published and have participated sufficiently to be accountable for all aspects of the work.

Funding This study is part of a research project supported by the Réseau Québécois de recherche en soins palliatifs et de fin de vie (Québec Research Network on Palliative and End-of-Life Care).

Competing interests None declared.

Patient consent for publication Not required.

Ethics approval This project was approved by the Research Ethics Committee of the CHU Sainte-Justine Research Center as a quality improvement clinical study (2018-1897). Participants provided written informed consent at the beginning of the online questionnaire.

Provenance and peer review Not commissioned; externally peer reviewed.

Data availability statement Data and additional information will be available upon reasonable request to the corresponding author

Open access This is an open access article distributed in accordance with the Creative Commons Attribution Non Commercial (CC BY-NC 4.0) license, which permits others to distribute, remix, adapt, build upon this work non-commercially, and license their derivative works on different terms, provided the original work is properly cited, appropriate credit is given, any changes made indicated, and the use is non-commercial. See: http://creativecommons.org/licenses/by-nc/4.0/.

ORCID iD

Claude Julie Bourque http://orcid.org/0000-0002-9407-0872

\section{REFERENCES}

1 Dupont-Thibodeau A, Langevin R, Janvier A. Later rather than Sooner: the impact of clinical management on timing and modes of death in the last decade. Acta Paediatr 2014;103:1148-52.

2 Verhagen AAE, Janvier A, Leuthner SR, et al. Categorizing neonatal deaths: a cross-cultural study in the United States, Canada, and the Netherlands. J Pediatr 2010;156:33-7.

3 Janvier A, Barrington K, Farlow B. Communication with parents concerning withholding or withdrawing of life-sustaining interventions in neonatology. Semin Perinatol 2014;38:38-46.

4 McCaffrey M. Lives worth living. Issues Law Med 2017;32:215-24.

5 Ahlund S, Clarke P, Hill J, et al. Post-Traumatic stress symptoms in mothers of very low birth weight infants 2-3 years post-partum. Arch Womens Ment Health 2009;12:261-4.
6 Kim WJ, Lee E, Kim KR, et al. Progress of PTSD symptoms following birth: a prospective study in mothers of high-risk infants. J Perinatol 2015;35:575-9.

7 Picoraro JA, Womer JW, Kazak AE, et al. Posttraumatic growth in parents and pediatric patients. J Palliat Med 2014;17:209-18.

8 Janvier A, Lantos J, Aschner J, et al. Stronger and more vulnerable: a balanced view of the impacts of the NICU experience on parents. Pediatrics 2016;138:0655.

9 Challacombe FL, Salkovskis PM, Woolgar M, et al. A pilot randomized controlled trial of time-intensive cognitive-behaviour therapy for postpartum obsessive-compulsive disorder: effects on maternal symptoms, mother-infant interactions and attachment. Psychol Med 2017;47:1478-88.

10 Bourque CJ, Dahan S, Mantha G, et al. Improving neonatal care with the help of veteran resource parents: an overview of current practices. Semin Fetal Neonatal Med 2018;23:44-51.

11 Hall SL, Ryan DJ, Beatty J, et al. Recommendations for peer-to-peer support for NICU parents. J Perinatol 2015;35 Suppl 1:S9-13.

12 Dahan S, Bourque CJ, Reichherzer M, et al. Beyond a seat at the table: the added value of family stakeholders to improve care, research, and education in neonatology. J Pediatr 2019;207:e122:123-9.

13 Butler AE, Hall H, Copnell B. Ethical and practical realities of using letters for recruitment in bereavement research. Res Nurs Health 2017:40:372-7.

14 Stevens MM, Lord BA, Proctor M-T, et al. Research with vulnerable families caring for children with life-limiting conditions. Qual Health Res 2010;20:496-505.

15 Blume ED, Balkin EM, Aiyagari R, et al. Parental perspectives on suffering and quality of life at end-of-life in children with advanced heart disease: an exploratory study ${ }^{*}$. Pediatr Crit Care Med 2014;15:336-42.

16 Wool C, Kain VJ, Mendes J, et al. Quality predictors of parental satisfaction after birth of infants with life-limiting conditions. Acta Paediatr 2018;107:276-82.

17 Currie ER, Christian BJ, Hinds PS, et al. Life after loss: parent bereavement and coping experiences after infant death in the neonatal intensive care unit. Death Stud 2019;43:333-42.

18 Snaman JM, Kaye EC, Spraker-Perlman H, et al. Incorporating bereaved parents as faculty facilitators and educators in teaching principles of palliative and end-of-life care. Am J Hosp Palliat Care 2018;35:1518-25.

19 Bakhbakhi D, Burden C, Storey C, et al. PARENTS 2 Study: a qualitative study of the views of healthcare professionals and stakeholders on parental engagement in the perinatal mortality review -from 'bottom of the pile' to joint learning. BMJ Open 2018;8:e023792.

20 Janvier A, Bourque CJ, Dahan S, et al. Integrating parents in neonatal and pediatric research, 2018.

21 Creswell JW, Creswell JD. Research design: qualitative, quantitative, and mixed methods approaches. SAGE Publications, 2017.

22 Creswell JW, Klassen AC, Plano Clark VL, et al. Best practices for mixed methods research in the health sciences. Bethesda (Maryland: National Institutes of Health, 2013: 541-5.

23 Jackson K, Bazeley P. Qualitative data analysis with NVivo. 3rd ed. London: Sage, 2019.

24 Hsieh H-F, Shannon SE. Three approaches to qualitative content analysis. Qual Health Res 2005;15:1277-88.

25 Green J, Thorogood N. Qualitative methods for health research. SAGE Publications, 2009.

26 Miles MB, Huberman AM, Saldana J. Qualitative data analysis. Sage, 2013.

27 Guetterman TC, Fetters MD, Creswell JW. Integrating quantitative and qualitative results in health science mixed methods research through joint displays. Ann Fam Med 2015;13:554-61.

28 Akard TF, Duffy M, Hord A, et al. Bereaved mothers' and fathers' perceptions of a legacy intervention for parents of infants in the NICU. J Neonatal Perinatal Med 2018;11:21-8.

29 Linebarger JS, Sahler OJZ, Egan KA, et al. Coping with death. Pediatr Rev 2009;30:350-6.

30 Neonatal butterfly project. The butterfly project. Available: https:// www.neonatalbutterflyproject.org/about-us/ [Accessed 27 Mar 2019].

31 Richards J, Graham RH, Embleton ND, et al. Health professionals' perspectives on bereavement following loss from a twin pregnancy: a qualitative study. J Perinatol 2016;36:529-32. 